\title{
The role of peer-reviewed journals in esthetic surgery
}

\author{
Eun Sang Dhong, Ha Yoon Chung \\ Department of Plastic and Reconstructive Surgery, Korea University Guro Hospital, Seoul, Korea
}

Why should we write scientific papers? It is often thought that research manuscripts are written by scholars and professors at universities. Cosmetic surgeons in private practice often feel no need to publish papers. However, as doctors, we are scientists who practice medicine. Furthermore, the internet is flooded with medical information-and, all too often, misinformation. In this piece, as editors, we would like to discuss the importance of writing articles on esthetic plastic surgery to be published in peer-reviewed journals, with a focus on two perspectives.

\section{DIRECT-TO-CONSUMER PORTRAYALS OF ESTHETIC SURGERY}

We live in an age of advertising. All goods and services, including food, clothing, and shelter, are circulated and consumed based on advertisements. The same is true of the medical market in which we operate. The revision of the medical law in 2012 expanded the media where medical advertising is permissible to include the internet and transportation; as a result, one routinely sees hospital advertisements in city buses and subway stations. Advertising is particularly intense for cosmetic procedures, especially in areas like Sinsa-dong in Gangnam; for instance, if you go to the Gangnam Apgujeong subway station, you will see that it is plastered with plastic surgery advertisements. Mobile and online advertisements, including those on SNS and YouTube, have also been active recently. The problem with these advertisements is that they are posted without prior deliberation and approval by the Medical Advertising Review Committee of the Korean Medical Association.

For this reason, advertisements that are unfounded or not objectively verified are at least somewhat inappropriate for the public.

Received: Dec 3, 2020 Accepted: Dec 3, 2020

Correspondence: Eun Sang Dhong Department of Plastic and Reconstructive Surgery, Korea University Guro Hospital, 148 Gurodong-ro, Guro-gu, Seoul 08308, Korea

Tel: +82-2626-3135, Fax: +82-852-7484, E-mail: prsdhong@kumc.or.kr

Copyright $\odot 2021$ The Korean Society for Aesthetic Plastic Surgery.

This is an Open Access article distributed under the terms of the Creative Commons Attribution Non-Commercial License (https://creativecommons.org/licenses/by-nc/4.0/) which permits unrestricted non-commercial use, distribution, and reproduction in any medium, provided the original work is properly cited. www.e-aaps.org
Content that may dazzle consumers is being provided with no filter. In 2016, the Ministry of Health and Welfare conducted a comprehensive survey of online advertisements targeting the fields of plastic surgery, beauty, and obesity, and found that $27 \%$ of all advertisements violated the medical law [1].

The problem is that these advertisements directly target the consumer, which is known as direct-to-consumer advertising (DTCA). In some countries, including the United States, DTCA has led to negative impacts [2]. Excessive advertising expenditures by pharmaceutical companies, for which DTCA is permitted [3], promote inappropriate drug use for various problems [4], and the domestic market is being shaped by similar factors. Doctors invest efforts into advertising that improves the quality of medical services, whereas many of the advertisements targeting the public are unfounded. Critics have pointed out that this environment encourages unnecessary surgery. According to a survey conducted by the Consumer Protection Agency in 2014, 30\% of respondents answered that their choice of a medical institution for plastic surgery was affected by cosmetic advertisements. As such, advertisements have a considerable influence on patients' selection of a hospital or clinic.

Another problem is that consumers continue to believe that doctors who receive frequent media exposure are particularly skilled. Everyone advertises their good results, and there is a tendency to promote knowledge derived from one's own experience as an "expert opinion." The problem, however, is that there is no evidence supporting most of the content presented in advertisements. Since doctors are scientists, evidence must take the form of a written paper published in a peer-reviewed journal. Grounded claims and advertisements will appeal to the public; this is the first reason why we should write scientific articles.

\section{EVIDENCE-BASED ESTHETIC SURGERY}

According to health and medical statistics in 2018, the number of board-certified plastic surgeons was 2,379, and there were 954 plastic surgery clinics. The number of clinics that did not specify their major medical category increased from 4,954 in 2010 to 5,781 in 2018. Approximately one-third of these clinics are estimated to provide beauty treatments and plastic surgery in Seoul.

About 2,000 institutions in Gangnam, Seoul, are marketed as 
"plastic surgery clinics," but board-certified plastic surgeons operate only approximately $350-400$ of these clinics. Plastic surgery is indiscriminately performed by general practitioners or other specialists. Since doing so is not currently illegal, this social atmosphere is tolerated to some extent.

There are two problems with this situation. The first is that plastic surgery is being performed by general practitioners, or other specialists who have not received plastic surgery training. This is a behavior of territorial destruction that ignores the fact that plastic surgeons receive more than 4 years of training to develop the competencies necessary to perform specialized surgery. This may lead to a situation where surgeons have insufficient expertise to perform certain surgical procedures, posing a threat to patient safety. The second problem is the absence of peer-review. Numerous academic societies advocate for beauty or cosmetic surgery. However, these conferences often share information through seminars using audio-visual materials rather than published papers. Since most of this information has not been verified, it is highly likely to contain false and/or superficial knowledge. The fact that unverified information is being shared through numerous seminars raises the concern that patient safety may be adversely affected.

What should we do? Evidence-based medicine has become the standard internationally, and esthetic surgeons should also practice evidence-based medicine. In Korea, there are three evidence-oriented academic journals in plastic surgery: Archives of Aesthetic Plastic Surgery (AAPS; the official journal of the Korean Society for Aesthetic Plastic Surgery), Archives of Plastic Surgery, and Archives of Craniofacial Surgery. AAPS is the only evidence-based journal focusing on esthetic surgery with a peer-review system.

$A A P S$ can play an important role in solving the problems discussed above. First, AAPS can serve as a venue to publicize the long experience that Korean surgeons have accumulated in cosmetic plastic surgery, as more than half a century has passed since cosmetic surgery was launched as a field in Korea. Each person has his or her individual expertise and know-how, and the in-depth discussion forum known as peer-review will promote further development. AAPS is also a space where surgeons can share specialized knowledge related to Asian populations. As Western medicine forms the cornerstone of modern medical practice, most of the existing expertise is based on Westerners. However, there are differences between Westerners and Asians, ranging from racial differences such as anatomical characteristics and anatomical characteristics to culture and customs. By sharing knowledge and skills tailored to Asians, we can expect better results from cosmetic surgery. Second, experiences that have been peer-reviewed can serve as evidence, moving beyond only recognition by colleagues to support the practice of evidence-based medicine. Peer-reviewed studies in this field can help to address the problems caused by direct-toconsumer advertising, as discussed above, by strengthening the scientific basis of advertisements.

Additionally, peer-reviewed publications facilitate recognition of the originality of knowledge or skills. Consider the following hypothetical example: doctor A performed 1,000 surgical procedures and devised a new technique, while doctor B performed 20 cases after seeing and learning doctor A's technique at a conference and published the first article describing the technique. If so, the question arises of who can be recognized as the originator of this technique, or whether doctor B can be ethically censured. Ambiguity may occur because doctor A did not write a paper. If doctor A had written a paper describing the technique first and if the paper had been verified by colleagues, he or she would have been recognized for the originality of the technique. This is the second reason why we should write papers.

Science is like water. It flows and changes, and permeates from high to low. We must submit our own experiences in the form of articles, develop as academics, and be recognized. In an era full of advertisements, self-promotion without evidence is meaningless. Therefore, we should increase our own value by publishing peerreviewed articles. Accumulating evidence from clinical experience can pay off. Therefore, we must wisely take advantage of the opportunities provided by AAPS, a unique Korean journal focusing on esthetic plastic surgery, to become winners.

\section{NOTES}

\section{Conflict of interest}

Eun Sang Dhong is an editorial board member of the journal but did not involve in the peer reviewer selection, evaluation, or decision process of this article. No other potential conflicts of interest relevant to this article were reported.

\section{ORCID}

Eun Sang Dhong

Ha Yoon Chung

\section{REFERENCES}

1. Sportschosun. Plastic surgery hospitals in Gangnam are responsible for releasing the most "illegal advertisements" which mislead consumers [Internet]. Seoul: Sportschosun; c2016 [cited 2021 Jan 21]. Available from: https://sports.chosun.com/news/ntype.htm?id=201610130 100100170007113\&servicedate $=20161012$.

2. Gilbody S, Wilson P, Watt I. Benefits and harms of direct to consumer advertising: a systematic review. Qual Saf Health Care 2005;14:246-50.

3. Donohue JM, Cevasco M, Rosenthal MB. A decade of direct-to-consumer advertising of prescription drugs. N Engl J Med 2007;357:67381.

4. Wolfe SM. Direct-to-consumer advertising--education or emotion promotion? N Engl J Med 2002;346:524-6. 